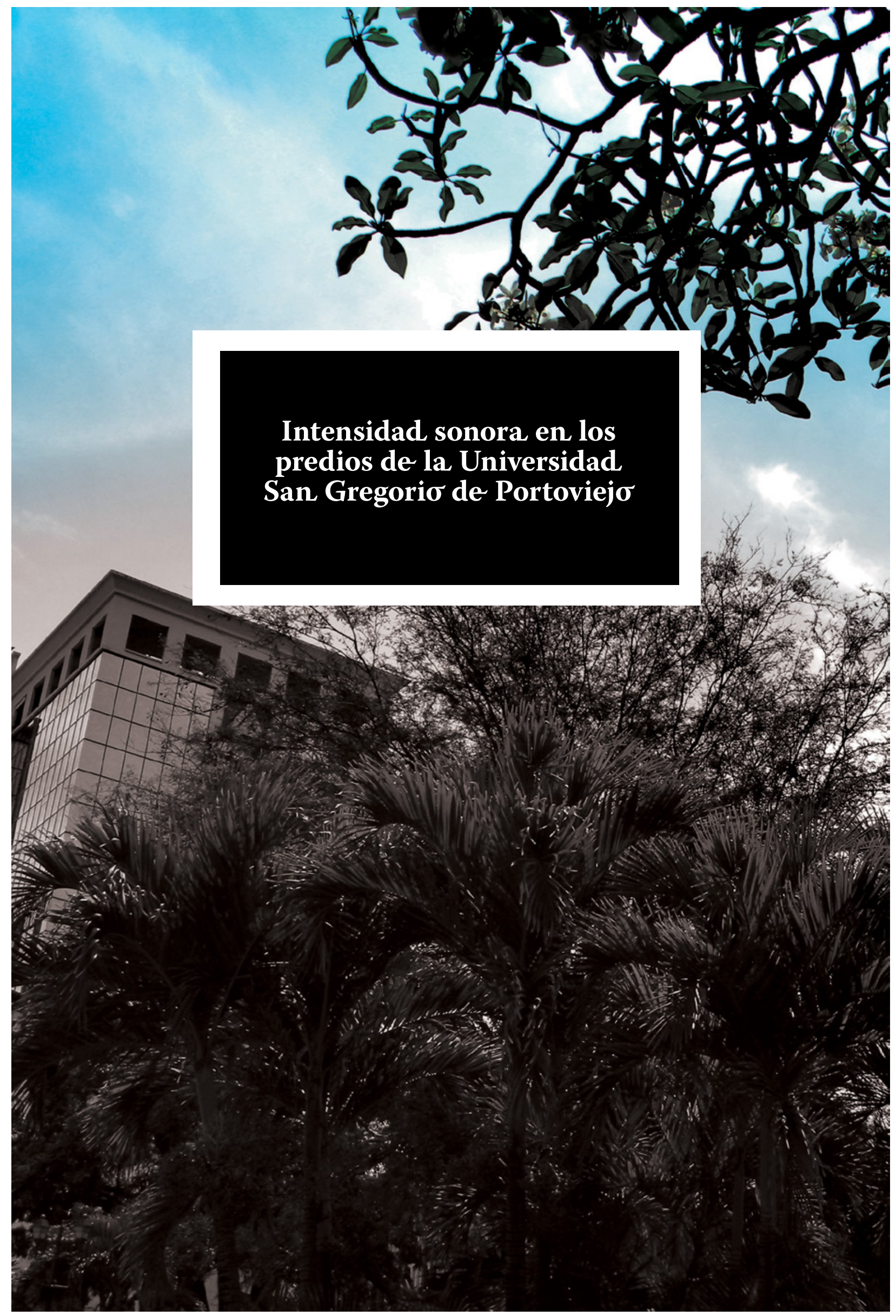




\title{
INTENSIDAD SONORA EN LOS PREDIOS DE LA UNIVERSIDAd SAN GREGORIO DE Portoviejo
}

\author{
Sound intensity in the premises of San Gregorio de Portoviejo \\ UNIVERSITY
}

\section{RESUMEN}

El propósito del presente trabajo es exponer los resultados de un estudio de contaminación sonora en los predios de la Universidad San Gregorio de Portoviejo, que no cuenta con investigaciones referentes a esta problemática ambiental. Para este análisis se tomó una muestra que abarca a los estudiantes de los últimos niveles de formación académica, docentes y empleados de la institución, mismos que estuvieron sometidos a estudios durante las horas de mayor concentración de vehículos y personal (principales generadores de ruido), además, se constató que existe desconocimiento sobre las normativas legales referentes a la contaminación sonora, y de cuáles son los límites permisibles, en general sobre todo en los centros de enseñanza.

PALABRAS CLAVE: Acústica; contaminación ambiental; intensidad sonora; ruido; salud.

Copyright @ Revista San Gregorio 2019. ISSN: 1390-7247; eISSN: 2528-7907.

(5)

\begin{abstract}
The purpose of this paper is to present the results of a sound pollution study on the premises of the San Gregorio de Portoviejo University, which does not have research related to this environmental problem. For this analysis, a sample was taken that includes the students of the last levels of academic training, teachers and employees of the institution, who were subjected to studies during the hours with the highest concentration of vehicles and personnel (main noise generators), In addition, it was found that there is ignorance about the legal regulations regarding sound pollution, and what are the permissible limits, in general, especially in educational centers.
\end{abstract}

KEYWORDS: Acoustic; environmental pollution; sound intensity; noise; health.

Copyright @ Revista San Gregorio 2019. ISSN: 1390-7247; eISSN: 2528-7907. ๑

\section{Darío Alberto Mendoza García}

소

Universidad San Gregorio. Portoviejo Manabí Ecuador

damega.100@gmail.com

\section{David Ernesto Moreira Moreira}

1.

Universidad San Gregorio. Portoviejo Manabi Ecuador

$\sim$
Adrián Eliceo Reyna García

م

Universidad San Gregorio. Portoviejo Manabí Ecuador



adrianreynag@gmail.com

ARTÍCULO RECIBIDO: 11 DE ABRIL DE 2019 


\section{INTRODUCCIÓN}

Cuando se habla de contaminación, inmediatamente se piensa en sustancias o gérmenes indeseados que se incorporan a un medio. En una acepción un poco más general, se puede hablar de contaminación cuando dicho medio es alterado nocivamente por cualquier agente. En este sentido, el ruido puede llegar a ser, efectivamente, un agente deletéreo, y por consiguiente puede ser un factor de contaminación ambiental (Miyara,1995).

La primera declaración internacional que contempló las consecuencias del ruido sobre la salud humana se remonta a 1972, cuando la Organización Mundial de la Salud (OMS) decidió catalogarlo genéricamente como un tipo más de contaminación. Siete años después, la Conferencia de Estocolmo clasificaba al ruido como un contaminante específico (Cattaneo \& Scrocchi, 2008).

El ruido se puede definir como un sonido no deseado o un sonido molesto e intempestivo que puede producir efectos fisiológicos y psicológicos en una persona o en un grupo de personas (Alonso, 2003, p.74).

De forma análoga, se pueden deducir los intervalos fuera de cuyos límites se producen sensaciones de molestias o incomodidad y también los niveles por encima de los cuales se producen trastornos en el sistema auditivo, y sus consecuencias en la salud de los individuos.

En la actualidad algunos estudios científicos y médicos demuestran el impacto negativo en el ser humano, tanto fisiológicos como psicosomáticos, causado por la generación de ruido en la audición, la salud y está considerado, como un agente contaminante invisible del mundo moderno, es un producto natural del desarrollo tecnológico y por consiguiente se debe normar y controlar.
El ruido ambiental es uno de los principales elementos de contaminación en las ciudades modernas. De acuerdo con el instituto del Ruido de Londres, los vehículos, con sus mecanismos, motores y el roce de los neumáticos con el pavimento, son los máximos responsables del ruido total en las grandes urbes, la necesidad de transportar personas y bienes de forma más rápida ha modificado la estructura de las ciudades, adaptándola al libre tránsito de medios de transporte masivo, particularmente el automóvil (Alfie \& Osvaldo, 2017).

La realidad es que, aun cuando parece que el ser humano se adapta al ruido, el oído nunca se cierra por lo que el cuerpo reacciona a este y se afecta, muchas veces sin que la anomalía sea detectada (López, 2009, p.3). Ver Tabla 1: Niveles críticos del ruido, 2007 (En Anexos).

Existen numerosas actividades que producen ruido como: el tráfico, las obras, las construcciones en general, el ocio, los procesos industriales, etc., que muchas veces se reflejan en daños causados a las personas, afectando su calidad de vida, por lo que se deben buscar alternativas de solución, que permitan reducir el impacto que generan estas actividades, sin renunciar a los beneficios y avances de la época actual.

El presente estudio plantea conocer los niveles de ruido y los efectos de la contaminación acústica en los predios de la Universidad San Gregorio de Portoviejo, efectuado en el mes de noviembre del 2017 para proponer actividades relacionadas con los aspectos referidos al ruido ambiental y la contaminación que este produce, resaltando el objetivo de realizar el mapa acústico del campus universitario, con el fin de concientizar y elevar los niveles de participación general activa de los universitarios, en el ámbito de la solución de los impactos ambientales que son provocados en el quehacer diario (Martínez, López, \& Ortíz, 2009) y evidenciar el cumplimiento de las normativas ambientales, municipales que regula esta afectación.

\section{METODOLOGÍA}

La metodología se basó en la recopilación, clasificación, análisis y definición de estudios complementarios, y la identificación preliminar del entorno y las personas a analizar, así como los elementos cartográficos, consulta a entidades, instituciones y organizaciones que 
manejan información concerniente al tema de estudio.

En esta investigación se utilizó un diseño exploratorio descriptivo, ya que es un tema poco estudiado en el entorno donde se desarrolló, se conocen estudios similares en otras latitudes, se realizaron preguntas a estudiantes, docentes y empleados que cumplen actividades en las instalaciones de la Universidad San Gregorio de Portoviejo, por otra parte, se determinó la intensidad del ruido, midiendo los niveles generados dentro y fuera de los predios, teniendo en cuenta que, como el ruido en los ambientes externos es fluctuante y procede de fuentes diversas se ha utilizado la geo estadística como herramienta para obtener valores representativos, hacer estimaciones, predicciones y simulaciones para evaluar alternativas de gestión del ruido. Adicionalmente se midieron los niveles de presión sonora expresados en la siguiente fórmula matemática:

$$
N P S e q=10 * \log * \sum(P i) 10^{\frac{N P S i}{10}}
$$

De los puntos seleccionados del área de estudio que comprende un poco más de 1 hectárea, se realizó una fragmentación determinando los hitos de mayor concentración de ruido: como los dos ingresos (Av. Metropolitana y Olímpica), el edificio administrativo, los 3 edificios destinados a las carreras universitarias y el teatro, en total 7 puntos que fueron georreferenciados, para posteriormente definir tres periodos de medición tomados en relación a las diferencias horarias y por la incidencia del tráfico vehicular estableciéndose de 09h00-10h00, 12h00-13h00, 16h0017 h00 con el empleo de un sonómetro marca Max Measure modelo MM-SMO1 Tipo 2, que cumple las normas internacionales como IEC651 y ANSI 1.4. Estos valores se compararon con los niveles permitidos establecidos en el TULSMA (Texto Unificado de Legislación Secundaria del Ministerio del Ambiente) de la legislación ambiental ecuatoriana, establecidos para los centros de enseñanza. (Decreto Ejecutivo 3516/2017).

Para hacer uso de los datos alcanzados se realizó una descarga de la información desde el sonómetro, que dispone de un software de comunicación, que permite descargar la información a un ordenador personal, todos los datos registrados y medidos en tiempo real en los horarios establecidos. El sonómetro se lo ubicó sobre un trípode a una distancia de 1,40m sobre el nivel del piso, el tiempo de medición fue de un minuto cuarenta segundos por cada punto y poder representar gráficamente esos datos para su correspondiente análisis. Con la ayuda de los sistemas de información geográfica se realizaron las interpolaciones para generar continuidad espacial de los niveles de ruido y ser representados a través de mapas acústicos.

Antes de la toma de datos en el campo se obtuvo la información climática de Portoviejo a través del INAMHI (Instituto Nacional de Meteorología e Hidrología, 2018).

Como parte de la metodología de investigación es necesario determinar la población universo a investigar (Universidad San Gregorio de Portoviejo), en su mayoría no se puede abarcar como objeto investigativo toda la población, es por esto que se recurre a un método estadístico de muestreo probabilístico para seleccionar una parte de toda la población que sea lo más representativo del colectivo, con las características relacionadas al estudio (docentes, estudiantes de los últimos niveles por su grado de permanencia en la institución y empleados de la universidad). Resulta primordial determinar el tamaño correcto de una muestra en donde se tomaron varios aspectos relacionados con los parámetros estimados, el sesgo, el error muestral, el nivel de confianza y la varianza poblacional (Rabolini,2009).

Muestra: Estudiantes de últimos niveles, docentes y empleados de la Universidad San Gregorio de Portoviejo, se utilizará la siguiente fórmula:

$$
\mathrm{n}=\frac{Z^{2} P Q N}{Z^{2} P Q+N e^{2}}
$$

$\mathrm{n}=$ Tamaño de la muestra

$\mathrm{Z}=$ Nivel de confiabilidad $=95 \%(1,96)$

$\mathrm{P}=$ Probabilidad de ocurrencia $=0,5$

$\mathrm{Q}=$ Probabilidad de no ocurrencia $1-0,5=0,5$

$\mathrm{N}=$ Población del centro de estudio $=420$ (estudiantes últimos niveles, docentes y personal administrativo) 
$\mathrm{e}=$ Error de muestreo $=5 \%(0,05)$

Muestra población centro de estudio

\section{MUESTREO ESTRATIFICADO}

$$
n=\frac{(1,96)^{2}(0,5)(0,5) 420}{(1,96)^{2}(0,5)(0,5)+420(0,05)^{2}}=\frac{403,37}{2,01}
$$

$$
n=200,68
$$

Es así, que de una población de 420 personas se obtuvo una muestra de 201.

La investigación se basó en la identificación de los requisitos bioéticos de la investigación y el análisis de las relaciones básicas existentes entre los principios bioéticos y las buenas prácticas, que en la actualidad señala (Borroto, 2015) es universalmente aceptado que existen dos principios inalienables para que una investigación sea moralmente valida: su utilidad social y su validez científica.

\section{RESULTADOS}

La Universidad San Gregorio de Portoviejo está ubicada en un sector de uso principal residencial de acuerdo al Plan de Ordenamiento Territorial de la ciudad de Portoviejo (2014) y que ha crecido urbanísticamente con la presencia de edificaciones residenciales, centros de educación primaria y secundaria, complejo deportivo, iglesias, empresas privadas, centros de diversión; que son parte del desarrollo y en cierta medida generadores de ruido que afectan a las actividades que se desarrollan en el entorno, su emplazamiento se genera específicamente en la parroquia Andrés de Vera, ocupando un área considerable limitada por cuatro vías, las Avenidas Metropolitana y Olímpica, como vías principales y sobre las que se generan los accesos y con otras dos calles adyacentes, la prolongación de la calle Kennedy y una calle sin nombre de escaso tráfico por su mal estado.

Una vez tomada la información se sigue el procedimiento requerido para el cumplimiento del objetivo final, que es la elaboración del mapa acústico en que se podrán observar las distintas zonas con sus niveles de presión sonora medida en decibeles ver Figura 1: Analizador de nivel de presión sonora (En Anexos).

Además, se emplearon gráficos de barras de dispersión en los monitores de ruido para obtención de resultados, ver la Figura 2: Localización de muestras de ruido. (En Anexos).

Como resultado de la pregunta número 1 ¿Conoce Ud., que la contaminación acústica es la segunda causa de origen ambiental que produce alteraciones en la salud humana según la OMS (Organización Mundial de la Salud)? Sobre el conocimiento que tiene el personal encuestado la mayoría se pronunció que si conocían con un 57,5\% que la contaminación acústica es la segunda causa de origen ambiental que produce alteraciones, en tanto un 42,5\% manifestó desconocer del tema.

En cuanto al resultado de la pregunta número 2 ¿Causa alguna dificultad o molestia en sus actividades cotidianas el ruido generado por el tráfico vehicular? Un 58\% de los encuestados manifestaron que, si les afecta el ruido generado por el tráfico vehicular, seguido de otro menor número que les afecta poco con un $37,50 \%$, en tanto que el 4,50\% manifestó que no siente ninguna molestia o dificultad.

La pregunta 3. ¿Qué grado de conocimiento posee sobre la contaminación sonora (provocada por el ruido) ?, Los encuestados aseguraron tener poco conocimiento sobre la contaminación sonora con un $63,50 \%$ y ninguno con un $11,50 \%$.

En tanto en la pregunta número 4 ¿Conoce Ud., los efectos que produce la exposición prolongada al ruido? Más de la mitad de los encuestados manifestaron que no conocían los efectos por exposición prolongada de ruido, con un $52,50 \%$.

La interrogante 5 ¿Sabia Ud., que existe una normativa legal que determina los niveles permisibles de ruido en los centros de enseñanza? Indudablemente deja evidenciado que se desconoce en su mayoría que existe una normativa legal que determina los niveles permisibles de ruido para los centros de enseñanza con un 57,50\%.

El cuestionamiento que se hace en la pregunta 6 ¿Durante su estadía o permanencia en el campus universitario, ha experimentado o ha sufrido alguna de las siguientes sensaciones? Un 75\% de los encuestados manifestó que en alguna ocasión ha sentido estrés e irritabilidad. 
La pregunta número 7 ¿Observa al interior de la Universidad San Gregorio de Portoviejo algún tipo de señalética o mapa acústico que identifique la contaminación por ruido? Se refleja que es casi nula la existencia de cualquier tipo de señalética que identifiquen la contaminación por ruido, aclarando de que actualmente no existe un mapa acústico.

En la última pregunta ¿Considera Ud., que la elaboración de un mapa acústico permitirá tomar acciones correctivas a las autoridades, personal administrativo, docentes y estudiantes para el control del ruido? La mayoría de los encuestados con un 52\% están convencidos que un mapa acústico lograría tomar ciertas acciones de orden correctivo para controlar el ruido y que deben ser emprendidas por las autoridades, aspecto que sería beneficioso para la comunidad universitaria.

Los resultados obtenidos por el sonómetro durante todo el mes de noviembre del 2017 en los 7 puntos establecidos en los predios de la universidad San Gregorio, demarcados se muestran en los Figuras 3, 4, 5 (Ver en Anexos).

\section{DISCUSIÓN}

Es relevante evidenciar que, aunque la mayor parte de la población universitaria tiene conocimiento acerca de la contaminación acústica, y tienen claro que es la segunda causa de origen ambiental que produce alteraciones en el comportamiento humano, no son capaces de medir la seriedad del tema, mientras que por otra parte, y con un nivel de problema aún mayor, la universidad no cuenta con las herramientas necesarias que muestren los puntos de mayor concentración de este tipo de contaminación, a través de un mapa de ruido. Este último es la representación cartográfica de los niveles de presión sonora (ruido) existentes en una zona concreta y en un periodo de tiempo determinado (Chávez, 2011).

Los autores reconocen la utilidad del mapa de ruido, para determinar la exposición de la población al ruido ambiental, para así adoptar los planes de acción necesarios para prevenir y reducir el ruido ambiental, en particular cuando los niveles de exposición puedan tener efectos nocivos en la salud humana.
De acuerdo con la OMS, un componente crucial de la implementación de planes para reducir el ruido, es tener un conocimiento cuantitativo razonable de la exposición a este, lo cual se logra a través de mapas de ruido. La OMS, a través de sus Guías para el ruido urbano, (1999, p.1) señala que, en la Unión Europea, alrededor de $40 \%$ de la población están expuestos al ruido de tránsito con un nivel equivalente de presión sonora que excede los 55 dBA por el día, y que el $20 \%$ están expuestos a más de $65 \mathrm{dBA}$. Si se considera la exposición total al ruido del tránsito, se puede calcular que aproximadamente la mitad de los europeos vive en zonas de gran contaminación sonora. Más de 30\% de la población están expuestos durante la noche a niveles de presión sonora que exceden $55 \mathrm{~dB}(\mathrm{~A})$ y que les trastornan el sueño. El problema también es grave en ciudades de países en desarrollo y se debe principalmente al tránsito.

La contaminación sonora se denomina a cualquier sonido que produzca malestar o que resulte excesivo en una determinada zona. Las personas que viven en las grandes ciudades sufren a menudo el ruido provocado por el tráfico de los coches, las bocinas de los autobuses y el paso de los trenes. Si sumamos a esto el sonido de los semáforos y el bullicio de los transeúntes se hace muy difícil, disfrutar del silencio o de sonidos agradables, llegando a sufrir patologías causadas por este malestar (Inspiraction, s.f.).

Delgado y Martínez (2015) en su análisis del ruido del área urbana de Cuenca relatan que la contaminación ambiental, identificada como emisiones al aire, ha ido en incremento en la ciudad de Cuenca, por causas que se le atribuye sobre todo al tráfico.

Entonces, es notorio que la población universitaria se encuentra expuesta constantemente a este tipo de contaminación, y en especial más específicamente, el ruido producido por los vehículos que transitan las calles y avenidas aledañas al campus, tanto así que, en la encuesta realizada, el 58\% de las personas manifestaron que el ruido vehicular les afecta e interfiere en la realización satisfactoria de sus actividades diarias.

La investigación realizada deja en evidencia que a pesar de que la mayoría de personas conocen que el ruido genera algún tipo de problema al comportamiento y desenvolvimien- 
to humano y al medio ambiente, es la misma cantidad que desconoce los efectos específicos que genera el ruido.

El ruido ambiental está asociado a múltiples actividades de los Campus, muchas de estas fundamentales, lo que ha provocado que las condiciones de ruido se lleguen a convertir o considerar como un elemento "natural, cotidiano o normal, es decir, algo con lo que irremediablemente se debe vivir...", otra acotación importante es que la percepción de ruido depende en gran medida del estado anímico, la sensibilidad individual, la atención, presión, concentración y el tipo de actividad que se está realizando, destacándose que cuando dicha actividad es obligatoria, supone una carga de estrés extra y una tendencia natural al aumento de sensibilidad y molestia (Martínez, López, \& Ortíz, 2009 (p.6).

Los efectos del ruido en las personas requieren de mayor estudio de forma sistemática y los resultados deben ser socializados para avanzar en la toma de conciencia, y así minimizar comportamientos de riesgo precursores de daño a partir de la exposición a altos niveles de ruido (Orozco \& González, 2015).

Ahora bien, la contaminación acústica no es algo que se queda solo en estadísticas, es también un asunto legal que incluso ha generado una ley nacional, que regula los niveles permisibles de ruido en los centros de educación, por ello por ejemplo en la pregunta $\mathrm{N}^{\circ} 5$ surge la siguiente interrogante ¿Sabía Ud., que existe una normativa legal que determina los niveles permisibles de ruido en los centros de enseñanza?, y las respuestas negativas sobrepasan las afirmativas, es decir que no se ha socializado lo suficiente la Ley por parte de las organizaciones competentes para ello.

\section{FUNDAMENTO LEGAL. LEY DE GESTIÓN AMBIENTAL}

La presente norma técnica es dictada bajo el amparo de la Ley de Gestión Ambiental y del Reglamento a la Ley de Gestión Ambiental para la Prevención y Control de la Contaminación Ambiental y se somete a las disposiciones de éstos, es de aplicación obligatoria y rige en todo el territorio nacional.

La presente norma técnica (Decreto Ejecutivo 3516/2017) determina o establece:
-Los niveles permisibles de ruido en el ambiente, provenientes de fuentes fijas.

-Los límites permisibles de emisiones de ruido desde vehículos automotores.

-Los valores permisibles de niveles de vibración en edificaciones.

-Los métodos y procedimientos destinados a la determinación de los niveles de ruido.

\section{CLASIFICACIÓN}

Esta norma establece los niveles máximos permisibles de ruido. La norma establece la presente clasificación:

1. Límites máximos permisibles de niveles de ruido ambiente para fuentes fijas

a. Niveles máximos permisibles de ruido de Ruidos

i. Medidas de Prevención y Mitigación

ii. Consideraciones generales

b. De la medición de niveles de ruido producidos por una fuente fija

c. Consideraciones para generadores de electricidad de emergencias

d. Ruidos producidos por vehículos automotores

e. De las vibraciones en edificaciones

Los niveles de presión sonora equivalente, NPSeq, expresados en decibeles, en ponderación con escala A, que se obtengan de la emisión de una fuente fija emisora de ruido, no podrán exceder los valores que se fijan en la Tabla 2, (Ver en Anexos).

El decibelio (db) es la unidad de medida del nivel de presión sonora, no es una unidad de medida absoluta, sino una unidad adimensional que expresa la diferencia entre dos niveles de intensidad y que es igual a 10 veces el logaritmo decimal de la relación entre una cantidad dada y otra que se toma como referencia (Durazno \& Peña , 2011).

Con los resultados obtenidos en esta investigación realizada en los predios universitarios, 
determinamos que los mayores problemas en la emisión de ruidos se concentran en las puertas de ingreso y salida (P1 y P2) en horas del mediodía a partir de las $12 \mathrm{~h} 00$, donde el mayor flujo de personas se moviliza, así lo reflejan las distintas mediciones mostrados en la figura 4, (Ver en Anexos) donde se supera el límite de exposición permisible, teniendo como fuente principal la generación de ruido que produce los automotores que circulan por la Av. Metropolitana y por la Olímpica.

En cuanto al ruido que se genera al interior de los predios, principalmente en el primer edificio de carrera (P4), se atribuye al generado por un cuarto de máquinas a una distancia aproximada de $20 \mathrm{~m}$ desde la coordenada del punto tomado. Así mismo dentro de los predios se generan ruidos cuando se concentran grandes grupos de estudiantes especialmente en los lugares destinados a descanso y actividades artísticas, como lo son las plazas, mismas que fueron puntos referentes para las mediciones realizadas.

A pesar de que existe una Ordenanza para la prevención y control de la contaminación ambiental originado por la emisión de ruidos del cantón Portoviejo en donde establece sanciones y disposiciones, niveles máximos de ruidos con controles esporádicos a través de su Departamento de Medio Ambiente, la urbe no cuenta con un mapa de ruido que indique los niveles acústicos de la ciudad (Ordenanza Municipal de Portoviejo, 2002).

\section{CONCLUSIONES}

-En el estudio realizado se ha logrado medir, representar y evaluar los niveles de ruido obtenido en diferentes puntos de la Universidad San Gregorio de Portoviejo, utilizando un sonómetro como equipo de medición sonora.

-De acuerdo al diagnóstico realizado queda demostrado que el tráfico vehicular externo afecta considerablemente a las actividades cotidianas al interior del centro de estudios.

-Es incipiente el conocimiento referente sobre contaminación sonora (ruido) y su efecto a la exposición prolongada.

-Existe desconocimiento sobre las normativas legales referentes a la contaminación sonora, cuales son los límites permisibles en general sobre todo en los centros de enseñanza.
-Se concluye que todos los valores registrados en los diferentes puntos tomados sobrepasan los límites permisibles determinados en la normativa legal en el TULSMA que establece $45 \mathrm{dBA}$ para la zona hospitalaria y educativa.

-La carencia de árboles en el entorno y hacia el interior de los predios tiene incidencia en la propagación del ruido

-Esta investigación será de gran utilidad para controlar y monitorear los niveles permisibles de ruido, hallando los puntos críticos y generando posibles soluciones para resolver la problemática.

\section{RECOMENDACIONES}

Se recomienda que, al continuar con la construcción de las demás edificaciones dentro de los predios universitarios, ya que aún faltan áreas por edificar se utilice o se emplee barreras disipadoras y absorbentes de ondas sonoras de tipo modular

Se sugiere realizar campañas de concientización sobre el problema de contaminación sonora

Se recomienda como medidas técnicas la aplicación de mecanismos como silenciadores en los escapes de los motores estacionarios y paredes aislantes de ruidos etc.

Se sugiere la aplicación de revestimiento poroso a las calzadas de bajo nivel de ruido, estos revestimientos reducen la generación y la propagación del ruido a través de una serie de mecanismos que pueden estar relacionados con la estructura abierta de la capa superior

Es recomendable que el GAD Municipal de Portoviejo realice mayores controles de ruido generado por el tráfico vehicular sobre todo en las dos arterias principales como son la avenida olímpica y metropolitana y que repercute hacia el interior del centro de enseñanza.

Se sugiere que las metodologías aplicadas en el presente estudio sean tomadas como referencias para futuros estudios en centros similares. 


\section{REFERENCIAS BIBLIOGRÁFICAS}

Alfie Cohen, Miriam, \& Salinas Castillo, Osvaldo, (2017). Ruido en la ciudad. Contaminación auditiva y ciudad caminable. Estudios demográficos y urbanos, 32(1), 6596.

Alonso, A. D. E. (2003). Contaminación acústica y salud Noise pollution and health. Observatorio medioambiental, 6, 73-95.

Borroto Cruz , E (2015). Bioética e investigación : Puente hasta el presente y para futuro. Revista San Gregorio , 6-15

Cattaneo, M., Vecchio, R., López, M., Navilli, L., \& Scrocchi, F. (2008). Estudio de la contaminación sonora en la ciudad de Buenos Aires.

Chávez, K. (2011). Anáisis multivariable de la afectación en terminos de ruido y demoras debido al control del tráfico en carreteras. Cádiz, España.

Delgado, O. \& Martínez, J. (2015). Elaboracion del mapa de ruido del area urbana de la ciudad de Cuenca-Ecuador , empleando la tecnica de interpolación geoestadistica Kriging ordinario.

Durazno Moscoso Saul \& Peña Durán Diego. (2011). Influencia de las Actividades Humanas Cotidianas en la Contaminación Acústica de la Zona de Regeneración Urbana de la ciudad Cuenca.(Tesis .Ing Ambiental). Universidad Politécnica Salesiana Cuenca .268P. Cuenca, Ecudor.

Decreto Ejecutivo 3516/2017, de última modificación: 29-mar.-2017, Texto Unificado de Legislación Secundaria del Ministerio del Ambiente. Libro VI, Anexo 5. Límites Permisibles de Niveles de Ruido Ambiente para fuentes fijas y móviles, y para vibraciones. Edición Especial 2 de 31-mar.-2003.

Inspiraction. (s.f.). INSPIRACTION. Obtenido de https:// www.inspiraction.org/cambio-climatico/contaminacion/ tipos-de-contaminacion/contaminacion-sonora
Instituto Nacional de Meteorología e Hidrología (Noviembre de 2018). INAMHII. Obtenido de http:// www.serviciometeorologico.gob.ec/pronostico-deltiempo-y-productos/

López, A. (2009). Intensidad de Ruido a la que se exponen los maestros en una escuela superior de la región central de puerto rico y su percepción al respecto, Tesis, San juan, Puerto Rico

Martínez, J., López, J., \& Ortíz, J. (2009). El Entorno Acústico en los Centros Universitarios: Análisis y Propuestas. 6.

Miyara, F. (1995). Contaminación acústica urbana en Rosario. Universidad Nacional de Rosario.

Ordenanza Municipal de Portoviejo. (expedida el 19 de agosto del 2002). Ordenanza Codificada para la Prevención y Control de la Contaminación Ambiental originado por la Emisión de Ruidos. Portoviejo.

Organización Mundial de la Salud. (1999). Guia para el Ruido Urbano. Basado en el documento "Community Noise"(version española). Londres Reino Unido.

Orozco Medina, M., \& González, A. (2015). La importancia del control de la contaminación por ruido en las ciudades. Ingeniería, 19 (2), 129-136.

Plan de Ordenamiento Territorial de Portoviejo. Gobierno Autónomo Descentralizado del cantón Portoviejo (2014).Cantón Portoviejo. República del Ecuador

Rabolini, N. M. (2009). Técnicas de muestreo y determinación del tamaño de la muestra en investigación cuantitativa. Revista argentina de humanidades y ciencias sociales, 2 . 
ANEXOS

\begin{tabular}{|c|l|}
\hline Nivel de ruido (db) & \multicolumn{1}{|c|}{ Efectos nocivos } \\
\hline $\mathbf{3 0}$ & $\begin{array}{l}\text { Dificultad en conciliar el sueño y perdida en la } \\
\text { calidad del descanso nocturno }\end{array}$ \\
\hline $\mathbf{4 0}$ & Dificultad en la comunicación verbal \\
\hline $\mathbf{4 5}$ & Probable interrupción del sueño \\
\hline $\mathbf{5 0}$ & Malestar diurno moderado \\
\hline $\mathbf{5 5}$ & Malestar diurno fuerte \\
\hline $\mathbf{6 5}$ & Comunicación verbal extremadamente difícil \\
\hline $\mathbf{7 5}$ & Perdida de oído a largo plazo \\
\hline $\mathbf{1 1 0 - 1 4 0}$ & Perdida de oído a corto plazo \\
\hline
\end{tabular}

Tabla 1: Niveles críticos del Ruido, 2007. Para sonidos impulsivos, valores dependientes de la duración del sonido y del número de exposiciones al mismo

Fuente: http://www.sistemasynkro.com/docs/Efectos_del_ruido_sobre_la_salud_la\%20sociedad_y_la_econom\%C3\%ADa.pdf

\begin{tabular}{|l|c|c|}
\hline \multirow{2}{*}{$\begin{array}{c}\text { TIPO DE ZONA SEGÚN EL } \\
\text { USO DE SUELO }\end{array}$} & \multicolumn{2}{|c|}{$\begin{array}{r}\text { NIVELES DE PRESIÓN SONORA } \\
\text { EQUIVALENTE NPSeq Db(A) }\end{array}$} \\
\cline { 2 - 3 } & DE 06:00 A 20:00 & DE 20:00 A 06:00 \\
\hline Zona hospitalaria y educativa & 45 & 35 \\
Zona residencial & 50 & 40 \\
Zona residencial mixta & 55 & 45 \\
Zona comercial & 60 & 50 \\
Zona comercial mixta & 65 & 65 \\
Zona industrial & 70 & 60 \\
\hline
\end{tabular}

Tabla 2: Niveles Máximos de Ruido Permisible según Uso de Suelo

Fuente: Texto Unificado de Legislación Secundaria del Ministerio del Ambiente. Libro VI Anexo 5 (Tabla 1). (Decreto Ejecutivo 3516/2017). 
ANEXOS

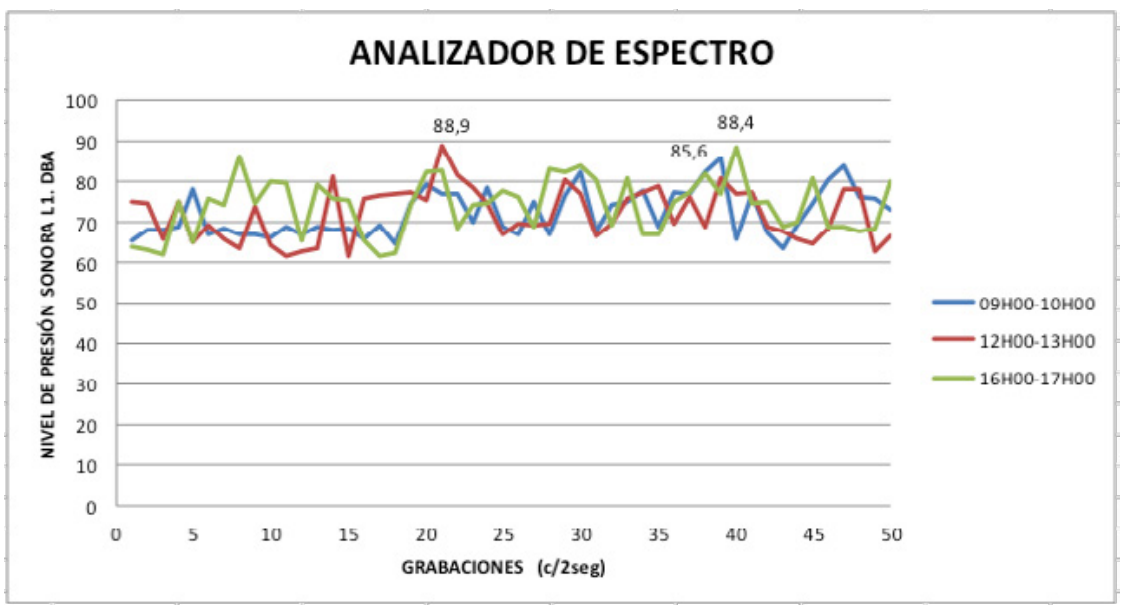

Figura 1: Mediciones Punto 1

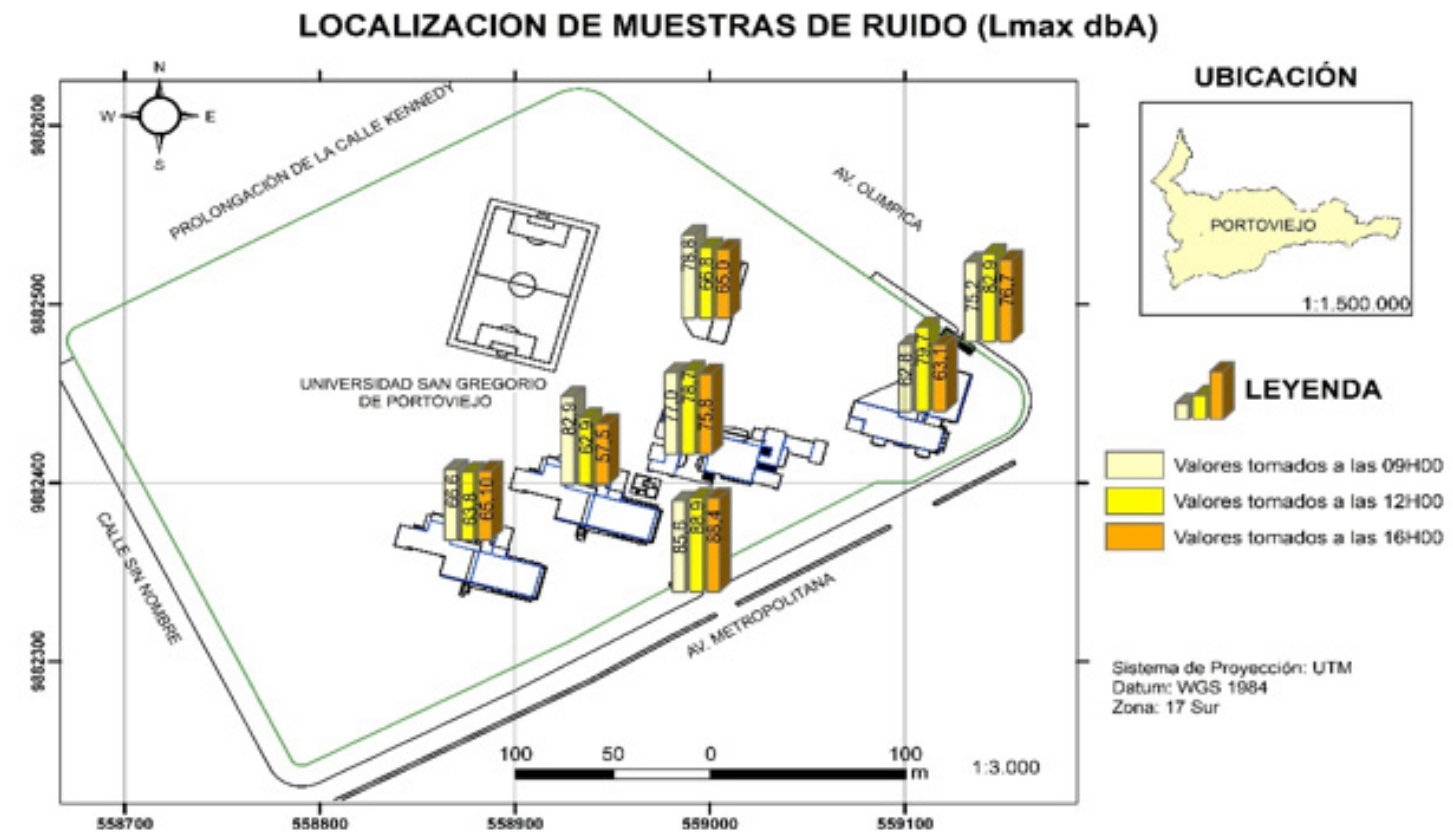

Figura 2: Localización de muestras de ruido 


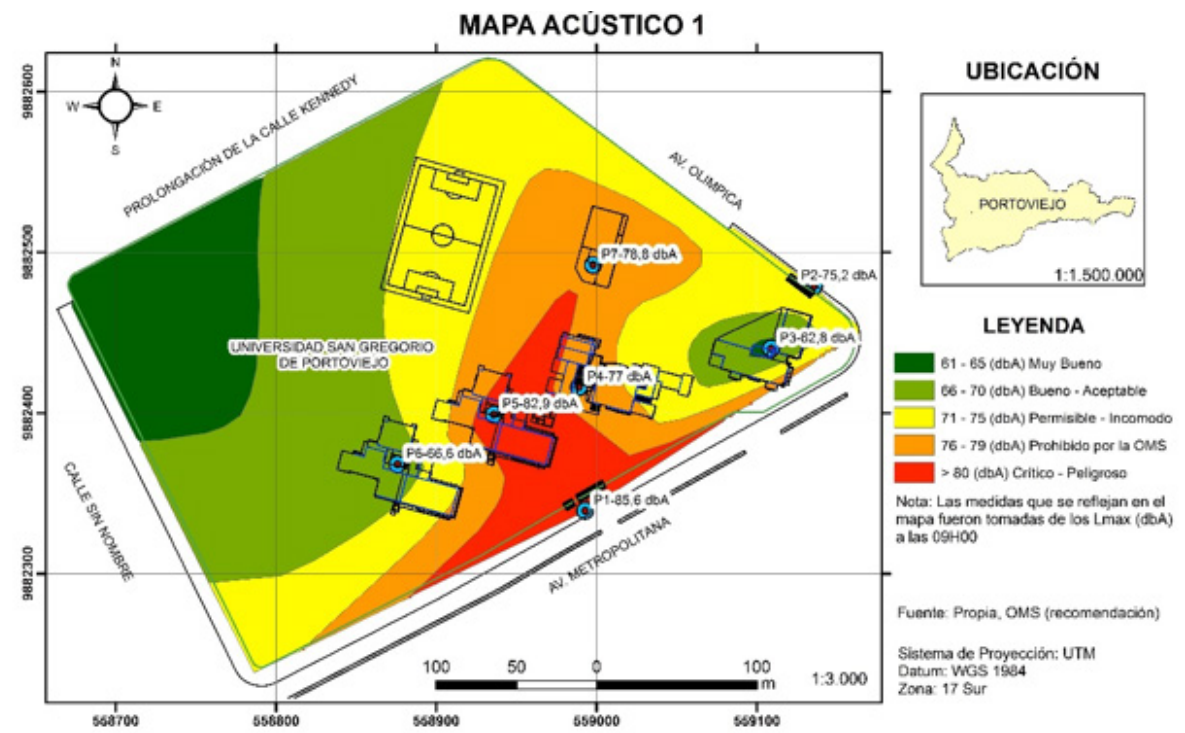

Figura 3: Mapa Acústico 1
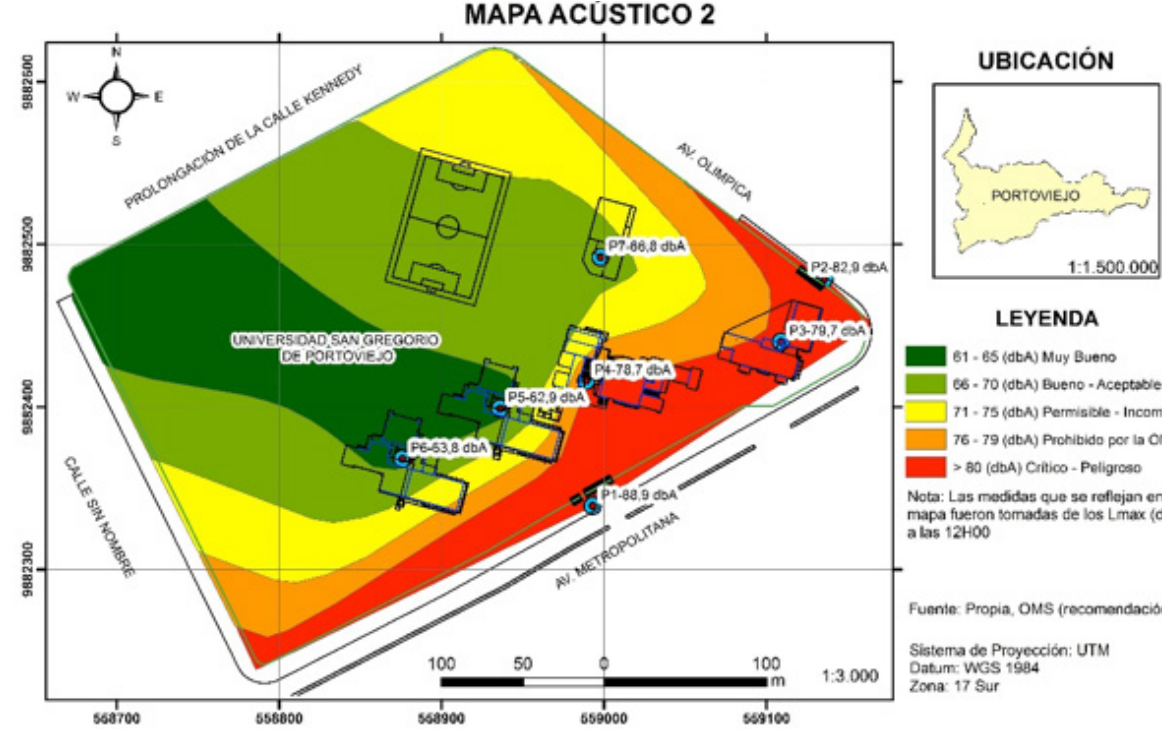

\section{LEYENDA}

51 - 65 (dba) Muy Bueno 166 - 70 (dDA) Bueno - Aceptabie 71.75 (dba) Permiside - Incomodo 76 - 79 (dba) Prohibido por la OMS 1 $>80$ (dbA) Critico - Peligrose Nota: Las modidas que se reflejan en el
mapa fueron somadas de los Lmax (ctaA) mapa tueron

Fuente: Propia, OMS (recomendación) Sistema de Proyección: UTM Datum: WGS
Zona: 17 Sur

Figura 4: Mapa Acústico 2
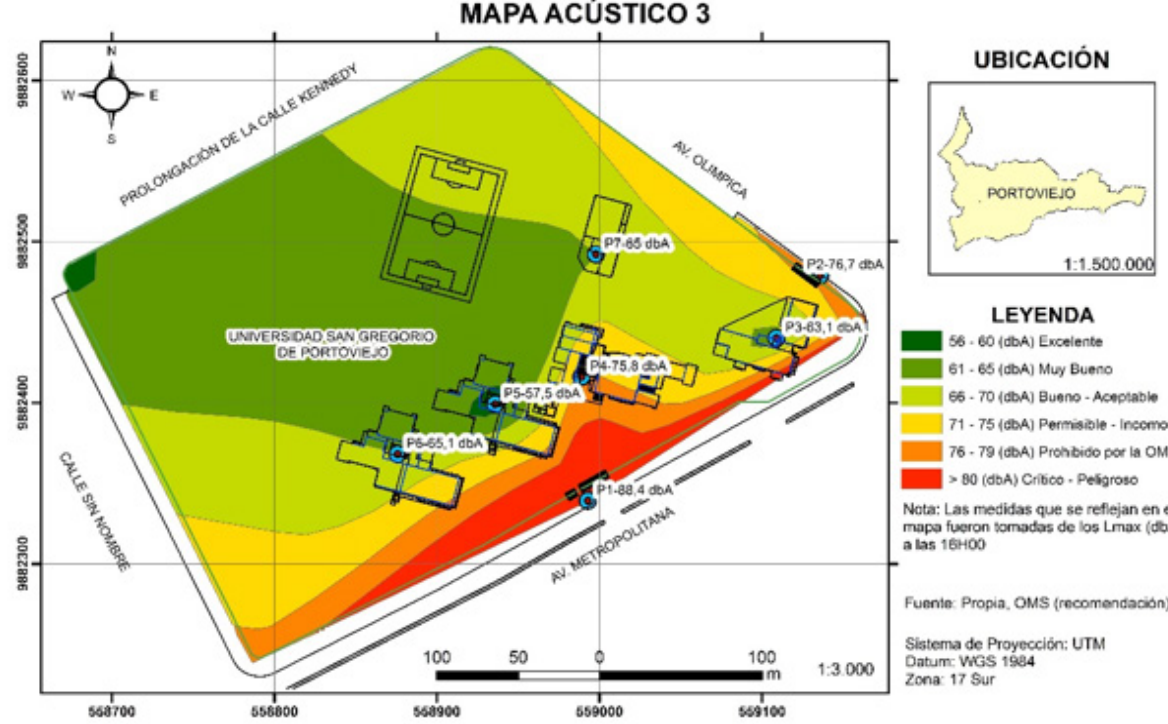

\section{LEYENDA}

56 - 60 (diba) Ercelente 65 - 70 (dba) Bueno - Aceplatio

71 - 75 (gha) Permisibile - incomode 76 - 79 (Eha) Pronbico por la OMS $>80$ (ADA) Cribco - Peligroso Nota: Las medidas que se reflejan en el mapa tueron

Fuente: Propia, OMS (recomendacion) Sistema de Proyecciōn: UTM 569700 500900 550000

Figura 5: Mapa Acústico 3 